\title{
Histochemistry and Angiogenesis in Thyroid Gland of Prenatal Indian Buffalo
}

\author{
Jatinderveer Kaur Billing ${ }^{1}$, Opinder Singh ${ }^{1 *}$, Devendra Pathak ${ }^{1}$ and R.S. Sethi ${ }^{2}$ \\ ${ }^{1}$ Department of Veterinary Anatomy, Guru Angad Dev Veterinary and Animal Sciences University, Ludhiana, Punjab, INDIA \\ ${ }^{2}$ Department of Animal Biotechnology, Guru Angad Dev Veterinary and Animal Sciences University, Ludhiana, Punjab, INDIA
}

*Corresponding author: O Singh; E-mail: singhopinder68@gmail.com

Received: 27 Aug., 2021

Revised: 23 Sept., 2021

Accepted: 28 Sept., 2021

\begin{abstract}
The present study was conducted on buffalo foetuses $(\mathrm{n}=19)$ ranging from 7.4 (62 days) to $108 \mathrm{~cm}$ (317 days) curved crown rump length (CVRL) to elucidate distribution of various histochemical moieties. The foetuses were divided into three groups based on CVRL. The content of neutral mucopolysaccharides was weak to moderate in Group I in parenchyma. The basement membrane of follicles was Periodic Acid Schiff (PAS) positive with colloid material in few follicles indicating the beginning of appearance of colloid containing neutral mucopolysaccharides. The developing capsule in Group I showed positive reaction for acid mucopolysaccharides. The follicular epithelium showed mixed reaction for mucopolysaccharides. In Group 2 connective tissue strands in the interfollicular space were positive for acid mucopolysaccharides. Intrafollicular colloid showed differential moderate to strong reaction for positive for neutral mucopolysaccharides and resorption vacuoles appeared at this stage. In Group III colloid was intensely PAS positive whereas follicular epithelium showed mixed reaction for mucopolysaccharides. The capsule was intensely positive for acid mucopolysaccharides. The lipid content was weak in all the groups, Protein content showed a progressive increase with increase in gestational age. Immunohistochemical studies on angiogenesis revealed progressive increase in number and size of blood vessels along with gestational age. The distribution of various histochemical moieties and angiogenesis reflected the physiological and development requirements during prenatal development.
\end{abstract}

\section{HIGHLIGHTS}

(0 Immunohistochemical markers were used to study the angiogenesis in prenatal thyroid of buffalo.

(0 The distribution of histochemical moieties and angiogenesis were correlated with physiological and developmental requirements.

Keywords: Histochemistry, Angiogenesis, Thyroid, Prenatal, Buffalo

Thyroid gland is the first endocrine gland, to develop as a ventral- midline- endodermal diverticulum from the floor of foregut. It is primarily concerned with heat production and regulation of the body temperature as well as the adaptation to the surrounding environment. Rapid advances have been made in knowledge and understanding of thyroid in human (Fagman and Nilsson, 2010) due to its functional importance and various endocrine disorders such as congenital hypothyroidism and thyroid dysgenesis. The elucidation of various histochemical factors responsible for growth and development of thyroid gland in buffalo will be critical for an improved understanding of development and functions of the gland. So, the present study was conducted to elucidate distribution of various histochemical moieties in thyroid gland of buffalo during prenatal development.

\section{MATERIALS AND METHODS}

The present study was conducted on thyroid glands of buffalo fetuses $(n=19)$. The foetuses of different gestational age were obtained from pregnant non-descript buffaloes slaughtered at Gazipur Slaughter House, New Delhi and Veterinary Clinical complex, GADVASU, Ludhiana. After

How to cite this article: Billing, J.K., Singh, O., Pathak, D. and Sethi, R.S. (2021). Histochemistry and Angiogenesis in Thyroid Gland of Prenatal Indian Buffalo. J. Anim. Res., 11(05): 901-907.

Source of Support: None; Conflict of Interest: None 
the collection, the foetal body length was measured as curved line in centimeter with the help of inelastic thread along the vertebral column between the most anterior part of frontal bone to the rump at ischiatic tuberosity and designated as crown rump length (Edward, 1965).

The age of the foetuses was calculated by using the formula given by Soliman (1975).

$$
\begin{gathered}
Y=28.66+4.496 X(C V R L<20 \mathrm{~cm}) \\
Y=73.544+2.256 X(C V R L>20 \mathrm{~cm})
\end{gathered}
$$

Where $Y$ is age in days and $X$ is $C V R L$ in centimeters.

Based on CVRL the foetuses were divided into three groups. Group I comprised of foetuses of CVRL between 0-20 cm, Group II between $20.1 \mathrm{~cm}$ to $40 \mathrm{~cm}$ and Group III above 40 $\mathrm{cm}$. Immediately after collection tissue samples were fixed in ten per cent neutral buffered formalin (NBF) and liquid nitrogen to study the distribution of histochemical moieties in prenatal thyroid gland of buffalo. The NBF fixed tissue samples were processed for paraffin blocks preparation by acetone-benzene schedule (Luna, 1968) and sections of $5 \mu \mathrm{m}$ thickness were obtained on glass slides with rotary microtome. The paraffin sections were subjected to various histochemical staining methods viz. periodic acid Schiff (PAS) for neutral mucopolysaccharides (Sheehan and Hrapchak, 1973), periodic acid Schiff and alcian blue at $\mathrm{pH} 2.5$ for acid mucopolysaccharides, Bromphenol Blue for basic proteins (Chayen et al., 1969). The tissue samples fixed in liquid nitrogen were subjected to cryostat sectioning at $-20^{\circ} \mathrm{C}$ and $10-12 \mathrm{~mm}$ sections were obtained on glass slides for demonstration of lipids by Sudan black $\mathrm{B}$ method and phospholipids by acid hematin method (Chayen et al., 1969). Angiogenesis was localized using anti Von Willebrand factor primary antibody.

\section{RESULTS AND DISCUSSION}

\section{Histochemistry}

\section{Neutral and acid mucopolysaccharides}

At $7.4 \mathrm{~cm}$ CVRL (62 days), weakly PAS-positive parenchyma was surrounded by developing capsule. (Fig. 1). At $10.7 \mathrm{~cm}$ CVRL (77 days), parenchyma was moderately positive for neutral mucopolysaccharides. The basement membrane of follicles was PAS positive with colloid material in few follicles indicating the beginning of appearance of colloid containing neutral mucopolysaccharides at this age (Fig. 2). The parenchyma was surrounded by developing capsule which showed positive reaction for acid mucopolysaccharides. Ranjan et al. (2011) observed moderately PAS positive parenchyma surrounded by developing capsule at $13.7 \mathrm{~cm}$ CVRL in buffalo foetuses. At $13.5 \mathrm{~cm}$ CVRL ( 89 days), developing capsule was weakly positive for acid mucopolysaccharides and lumen of few follicles showed PAS positive reaction. The follicular epithelium showed mixed reaction for mucopolysaccharides (Fig. 4). El Shiekh et al. (1966) observed that the follicular epithelium showed mixed reaction for mucopolysaccharides in camel foetus. Ranjan et al (2011) observed that the capsule was moderate to strongly positive for acid mucopolysaccharide while the follicular epithelium showed mixed reaction for mucopolysaccharides in buffalo foetuses.
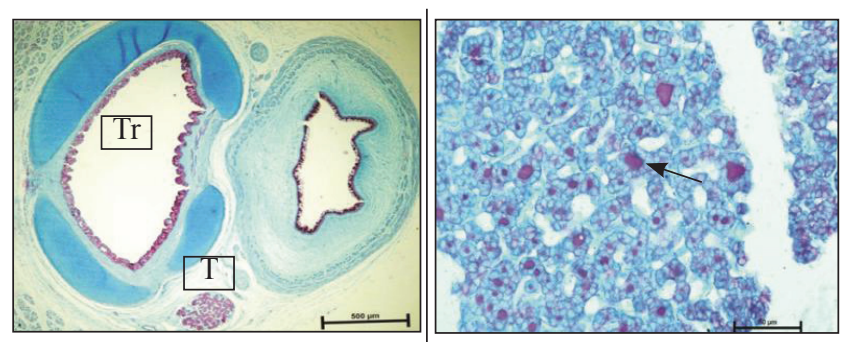

Fig. 1: Section of neck region Fig. 2: Section of thyroid of $7.4 \mathrm{~cm}$ CVRL (62 days) gland of $10.7 \mathrm{~cm} \mathrm{CVRL} \mathrm{(77}$ foetus showing PAS-positive days) foetus showing PASthyroid primordia $(\mathrm{T})$ on the positive colloid in few follicles lateral side of trachea (Tr) and (arrow). Follicular cells are oesophagus (O). PAS-AB X40 weakly positive for acid mucopolysaccharides. PAS-
AB X400

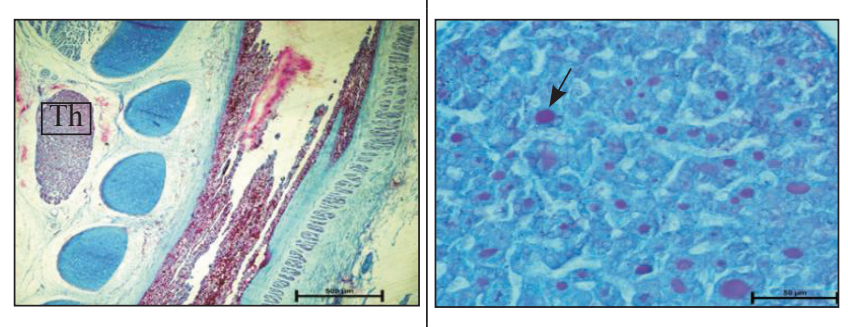

Fig. 3: Section of neck region of Fig. 4: Section of thyroid $13.5 \mathrm{~cm}$ CVRL (89 days) foetus gland of $13.5 \mathrm{~cm}$ CVRL (89 showing predominantly PAS- days) foetus showing PASpositive thyroid parenchyma positive colloid filled follicles (Th) surrounded by developing (arrow) surrounded by follicular capsule (arrow). PAS AB X40 cells showing mixed reaction for neutral and acid mucopolysaccharides. PAS-AB X400 
Intense PAS positive reaction was observed in the lumen of some follicles while mild reaction was observed in the others, indicating the increase in the content of colloid containing neutral mucopolysaccharides at $18.2 \mathrm{~cm} \mathrm{CVRL}$ (110 days) (Fig. 5).

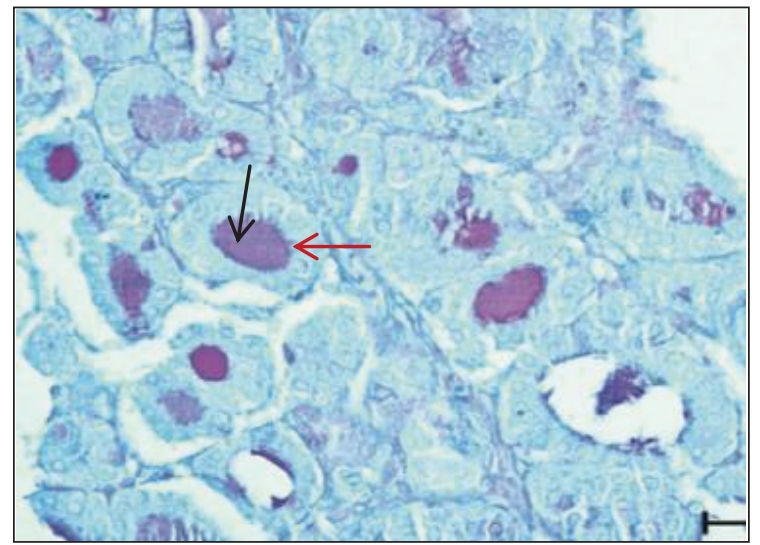

Fig. 5: Section of thyroid gland of $18.2 \mathrm{~cm}$ CVRL (119 days) foetus showing follicles filled with PAS-positive colloid (black arrow) and follicular cells showing mixed reaction for mucopolysaccharides (red arrow). PAS-AB X400

Capsule was moderately positive for acid mucopolysaccharide. Ranjan et al. (2011) observed strongly PAS positive colloid in some of the follicles at $20 \mathrm{~cm}$ CVRL. At $20.2 \mathrm{~cm}$ CVRL (119 days), connective tissue strands in the interfollicular space were positive for acid mucopolysaccharides (Fig. 6). Intrafollicular colloid was positive for neutral mucopolysaccharides and resorption vacuoles appeared at this stage. Banks (1992) mentioned that, the colloid was PAS-positive in domestic animals.

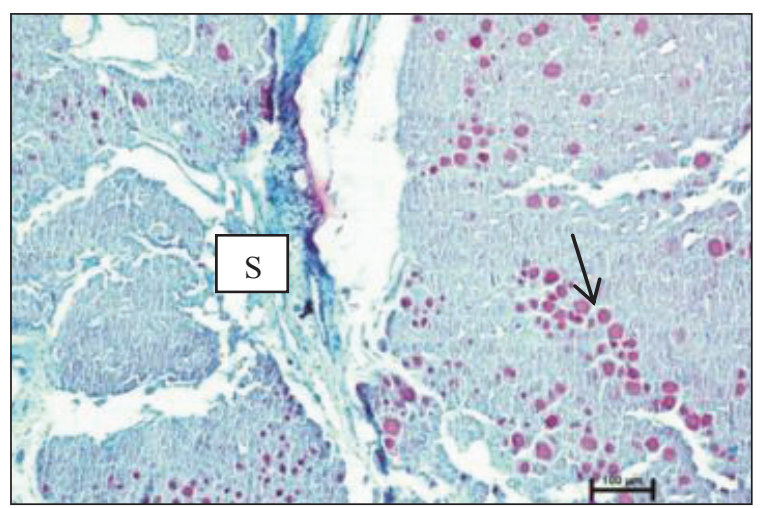

Fig. 6: Section of thyroid gland of $20.2 \mathrm{~cm}$ CVRL (119 days) foetus showing $\mathrm{AB}$-positive connective tissue septa $(\mathrm{S})$ and PAS-positive colloid in the follicles (arrow). PAS-AB X100
At $24 \mathrm{~cm}$ CVRL (128 days), large resorption vacuoles were observed in the intrafollicular colloid. El Shiekh et al. (1966) observed that the resorption vacuoles appeared at 70 days of gestation in intrafollicular colloid in camel foetus. The presence of colloid and resorption vacuoles was correlated with secretary activity of gland (Fig. 7).

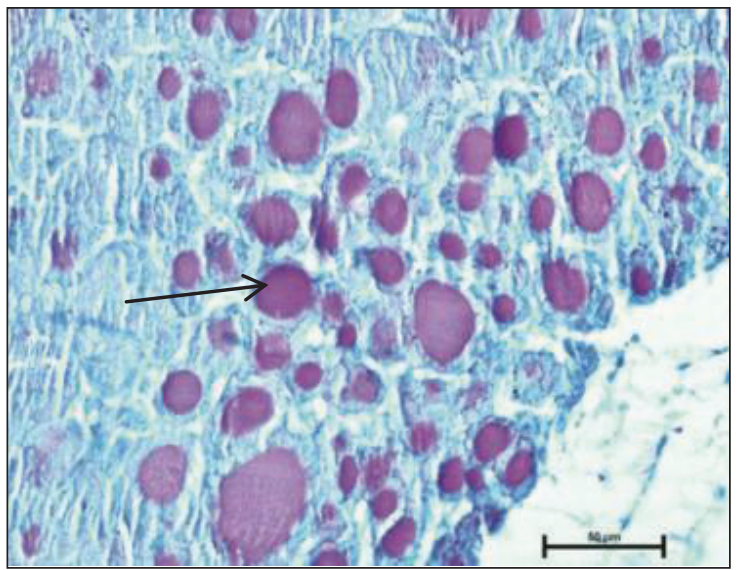

Fig. 7: Section of thyroid gland of $24 \mathrm{~cm}$ CVRL (128 days) foetus showing follicles filled with PAS-positive colloid (arrow)and lined by follicular cells showing mixed reaction for mucopolysaccharides. PAS-AB X400

Almost all follicles showed intense PAS positive reaction in lumen at $26.1 \mathrm{~cm}$ CVRL (132 days). Capsule was positive for acid mucopolysaccharides. At $31 \mathrm{~cm} \mathrm{CVRL}$ (143 days) mixed reaction was observed in follicular epithelium and intensely PAS-positive colloid (Fig. 8).

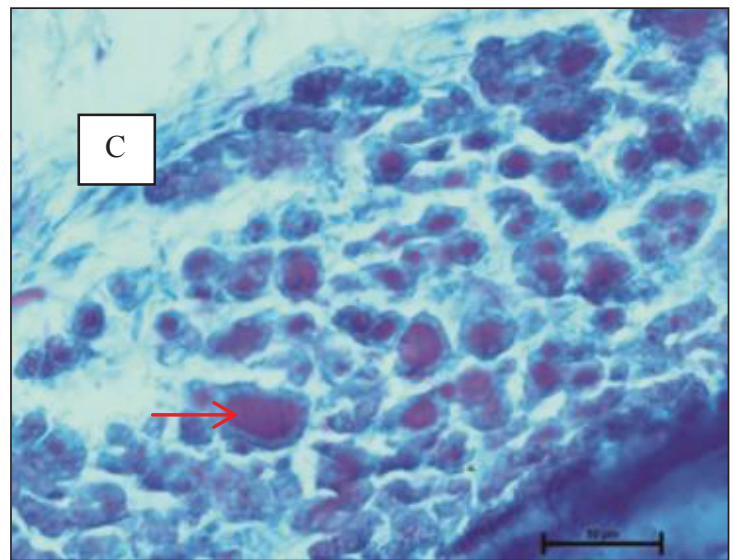

Fig. 8: Section of thyroid gland of $31 \mathrm{~cm} \mathrm{CVRL} \mathrm{(143} \mathrm{days)}$ foetus showing mixed reaction in follicular epithelium and intensly PAS-positive colloid (arrow). Capsule (C) is strongly positive for acid mucopolysaccharides. PAS-AB X400 
Capsule was strongly positive for acid mucopolysaccharides. At $50.8 \mathrm{~cm}$ CVRL (188 days), follicular cells showed mixed reaction with increased neutral mucopolysaccharides content (Fig. 9) Colloid was strongly positive for neutral mucopolysaccharides. At 108 cm CVRL (317 days), capsule is strongly positive for acid mucopolysaccharides and colloid in few follicles showing AB-positive reaction (Fig. 10). Similar content of neutral and acid mucopolysaccharides was observed during late stages of gestation.

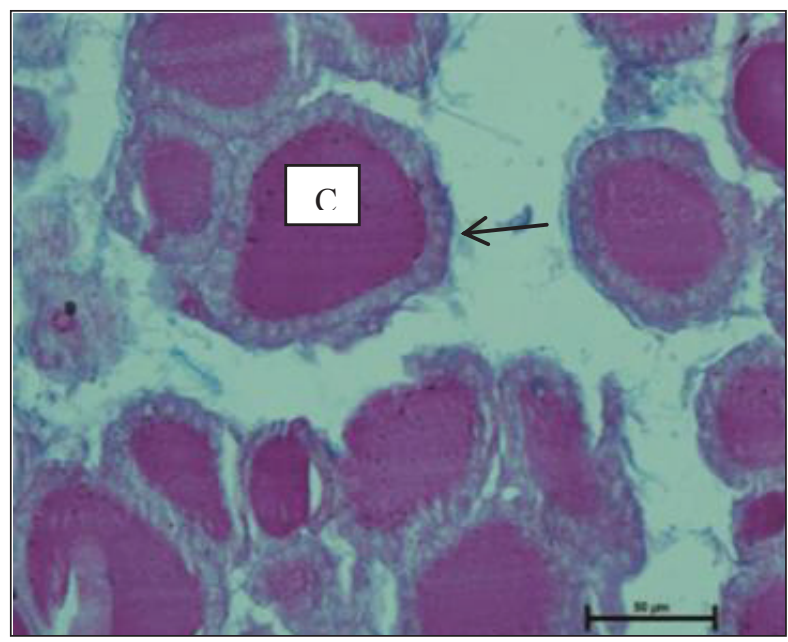

Fig. 9: Section of thyroid gland of $50.8 \mathrm{~cm}$ CVRL (188 days) foetus showing intensely PAS positive colloid (C) in all the follicles and weakly PAS-positive follicular cells (arrow). PASAB X400

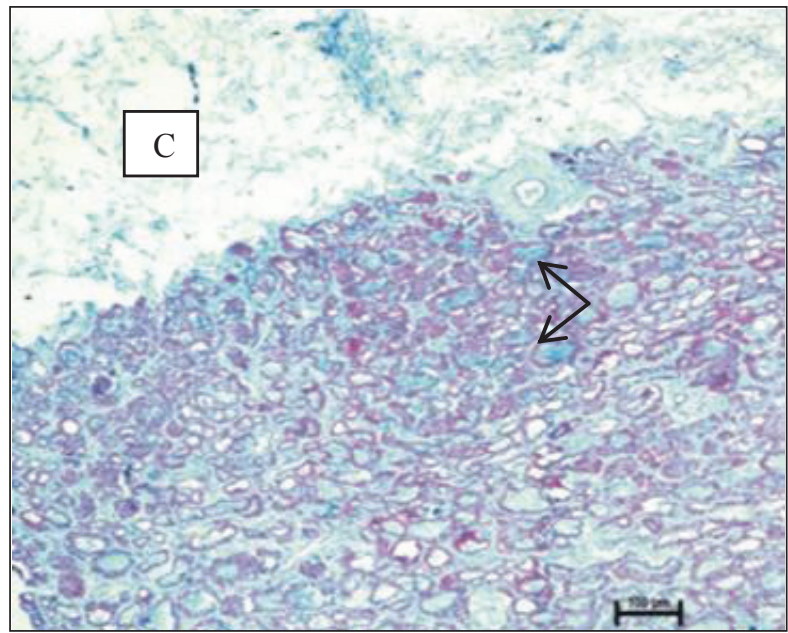

Fig. 10: Section of thyroid gland of $108 \mathrm{~cm}$ CVRL (317 days) foetus showing acid mucopolysaccharides in the capsule (C).
Colloid in some of the follicles showing acid mucopolysaccharide content (arrow). PAS-AB X100.

\section{Lipids}

The lipid content was weak in the capsule, follicular epithelium, intrafollicular colloid and interfollicular connective tissue in all the groups (Fig. 11\&12).

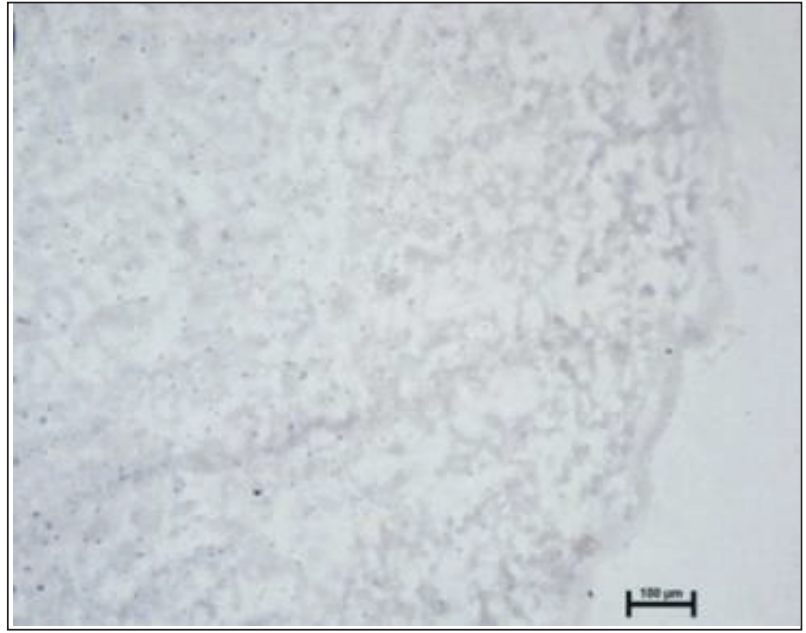

Fig. 11: Cryostat section of thyroid gland of $31 \mathrm{~cm}$ CVRL (143 days) foetus showing weak reaction for lipids in the parenchyma. Sudan Black B X100

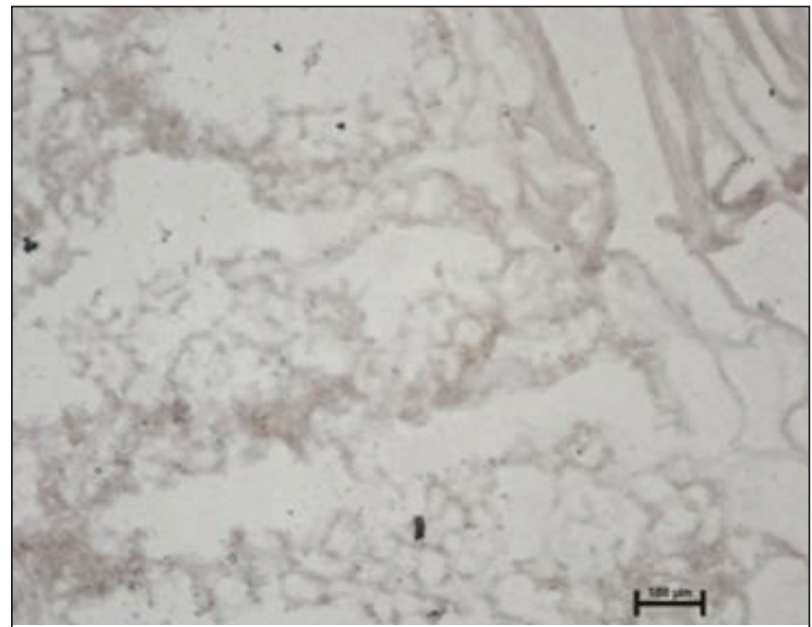

Fig. 12: Cryostat section of thyroid gland of $56 \mathrm{~cm}$ CVRL (200 days) foetus showing weak reaction for lipids in the parenchyma. Sudan Black B X100 


\section{Proteins}

The protein content was moderate in the capsule, parenchyma and interfollicular connective tissue in Group I (Fig. 13). The reaction was moderate to strong in Group II (Fig. 14). The protein content was maximum in capsule, parenchyma and interfollicuar connective tissue in Group III (Fig. 15 \&16).

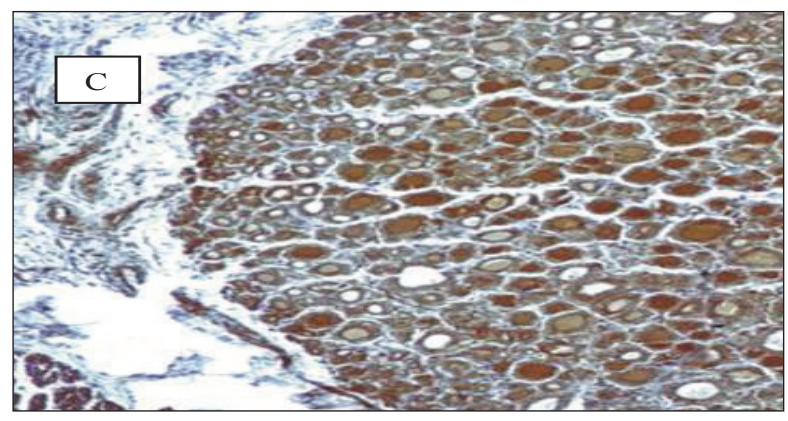

Fig. 13: Section of thyroid gland of $18.2 \mathrm{~cm}$ CVRL (110 days) foetus showing proteins in the capsule (C) and in the interfollicular spaces. Bromphenol blue X100

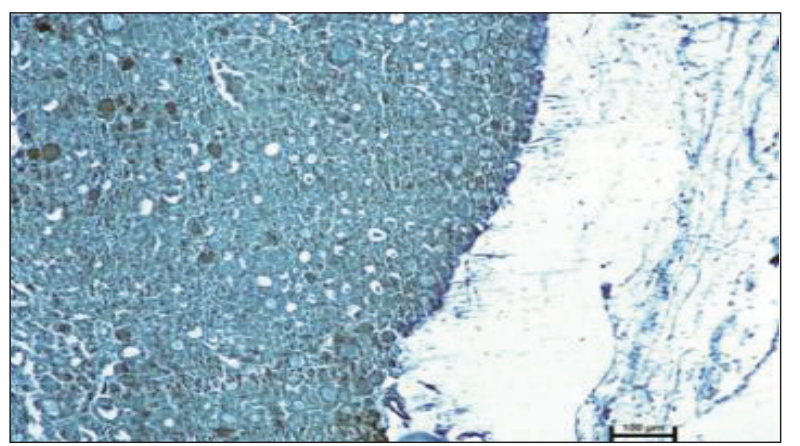

Fig. 14: Section of thyroid gland of $20.2 \mathrm{~cm}$ CVRL (119 days) foetus showing proteins in the capsule and parenchyma. Bromphenol blue X100

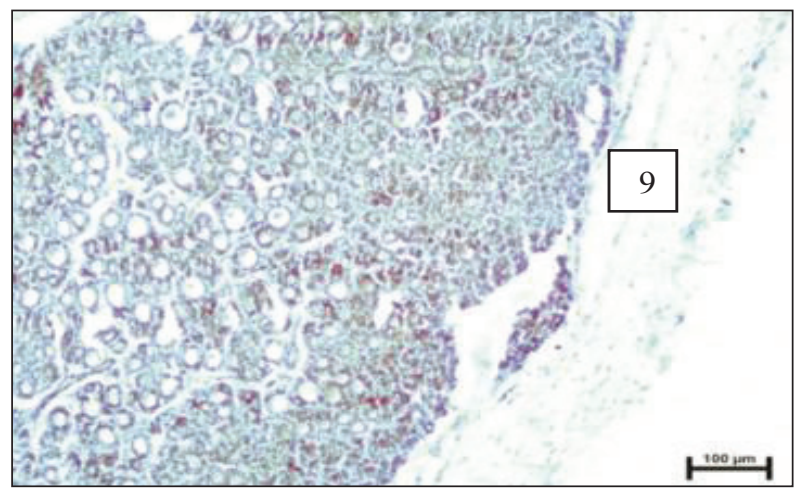

Fig. 15: Section of thyroid gland of $36 \mathrm{~cm}$ CVRL (155 days) foetus showing proteins in the capsule $(\mathrm{C})$ and in the parenchyma. Bromphenol blue X100

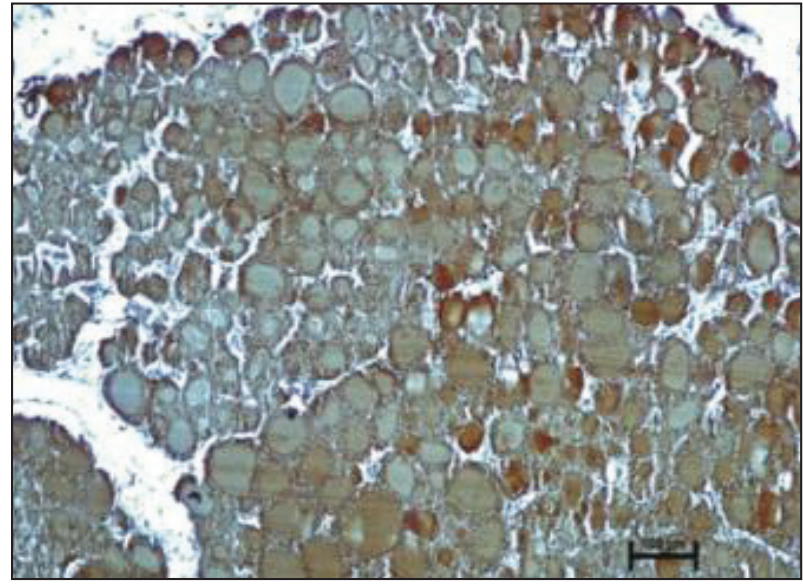

Fig. 16: Section of thyroid gland of $50.8 \mathrm{~cm}$ CVRL (188 days) foetus showing proteins in the parenchyma and interfollicular spaces. Bromphenol blue X100

\section{Angiogenesis}

Blood vessels were noticed in between the clumps of cells at $10.7 \mathrm{~cm}$ CVRL (77 days). At $13.5 \mathrm{~cm}$ CVRL (89 days) the thyroid primordia showed abundant blood vessels in the interfollicular space (Fig. 21). Hajovska (2002) noticed that the parenchyma of the thyroid gland, composed of solid cell networks, was irregularly divided by septa and was containing embryonal blood vessels in sheep foetuses during $32^{\text {nd }}$ to $36^{\text {th }}$ day of evolution.

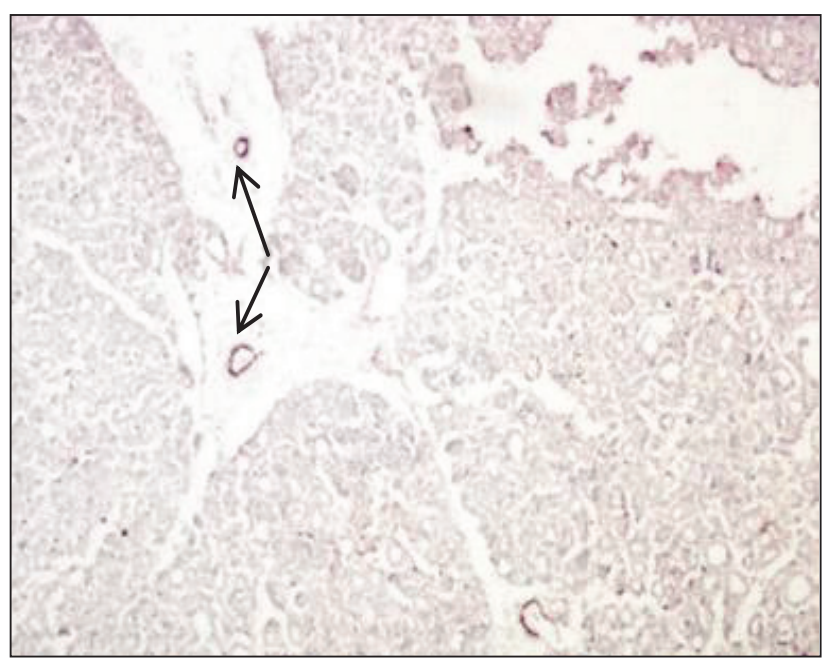

Fig. 17: Section of thyroid gland of $13.5 \mathrm{~cm}$ CVRL (89 days) foetus showing presence of blood vessels (arrow) in the interfollicular spaces. vWF X400 


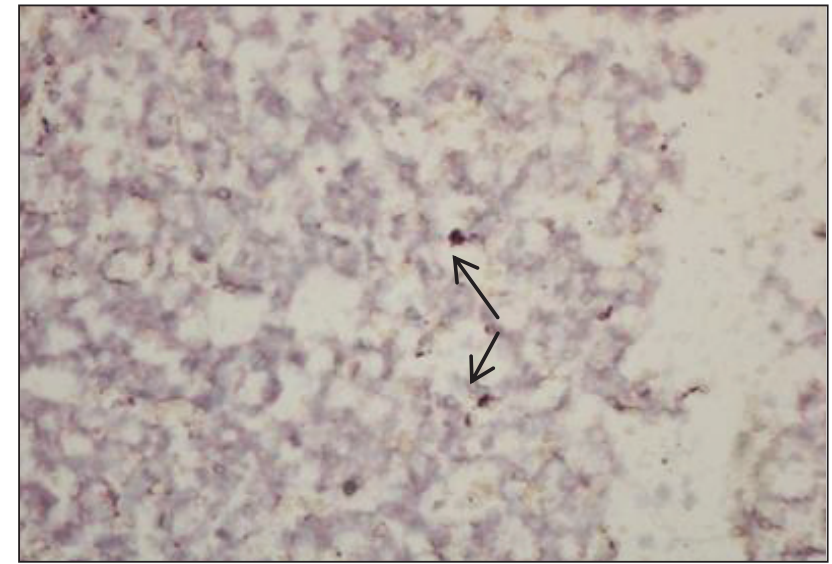

Fig. 18: Section of thyroid gland of $22 \mathrm{~cm}$ CVRL (123 days) foetus showing number of blood vessels (arrow) in the connective tissue. vWF X100

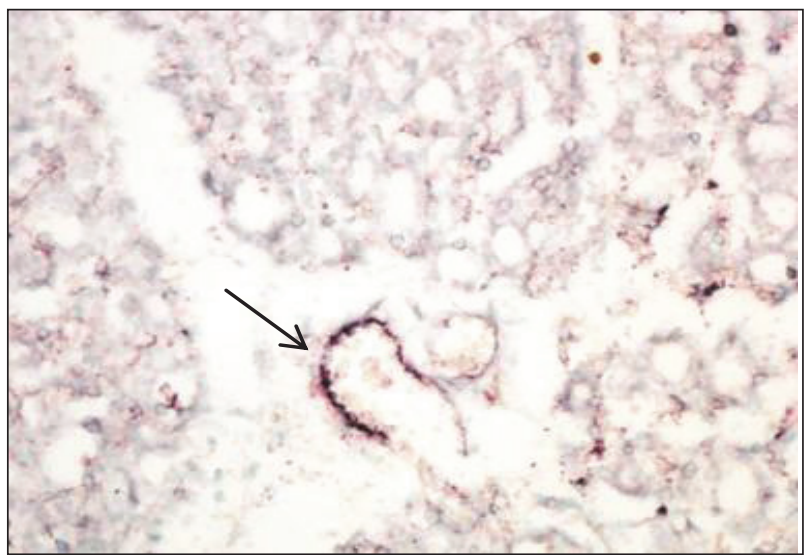

Fig. 19: Section of thyroid gland of $22 \mathrm{~cm}$ CVRL (123 days) foetus showing larger blood vessels (arrow) in the connective tissue. vWF X400

Ranjan et al. (2011) observed randomly arranged undifferentiated mesenchymal cells with large number of blood vessels upto $13.5 \mathrm{~cm}$ CVRL (90 days) (Fig. 17). At $18.2 \mathrm{~cm}$ CVRL (110 days), few large blood vessels were observed in the capsule. Ranjan et al (2011) observed compact epithelial strands separated by loose connective tissue with few blood vessels at $18.5 \mathrm{~cm}$ CVRL (112 days) buffalo foetus. At $22 \mathrm{~cm}$ CVRL (123 days), numerous blood capillaries were noticed in the connective tissue and intermingled in between the primitive follicles (Figs. 18 \& 19). Ramayya et al. (2012) noticed that the thyroid was highly vascular at $19.5 \mathrm{~cm}$ CVRL (115 days) in buffalo foetus. The number and size of blood vessels increased along with progression in gestation period. At $24 \mathrm{~cm}$ CVRL (128 days) blood vessels were observed in the interfollicular spaces (Fig. 20).

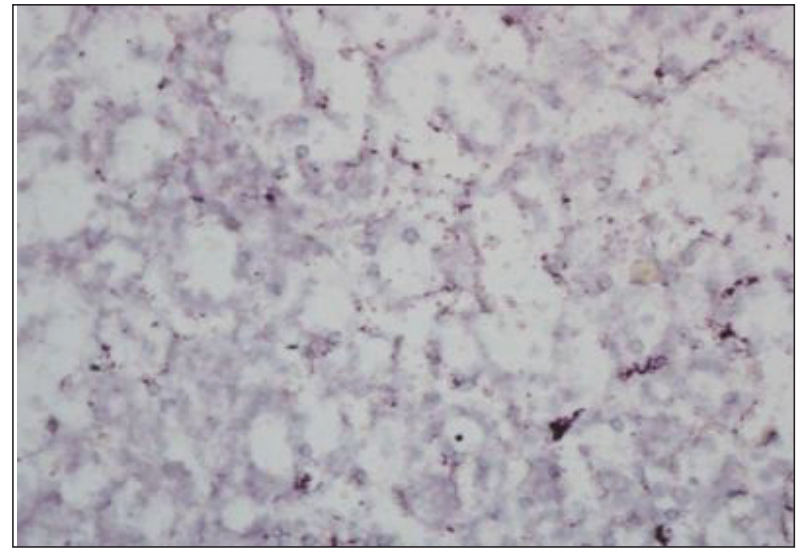

Fig. 20: Section of thyroid gland of $24 \mathrm{~cm}$ CVRL (128 days) foetus showing blood vessels in the interfollicular spaces. vWF $\mathrm{X} 400$

Numerous blood capillaries surrounded by connective tissue were found in the interfollicular spaces at $26.1 \mathrm{~cm}$ CVRL (132 days) (Fig. 21). These blood capillaries were lined by endothelial cells with elongated oval nucleus. At $50.8 \mathrm{~cm}$ CVRL (188 days), larger blood vessels were visible in the connective tissue in the interfollicular spaces (Fig. 22).

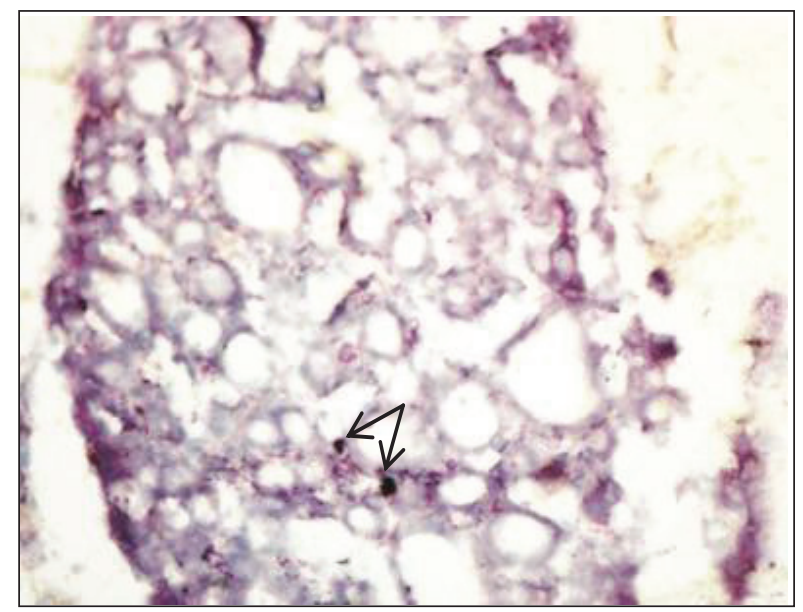

Fig. 21: Section of thyroid gland of $26.1 \mathrm{~cm}$ CVRL (132 days) foetus showing increase in the number of blood vessels (arrow) in the interfollicular spaces. vWF X400 


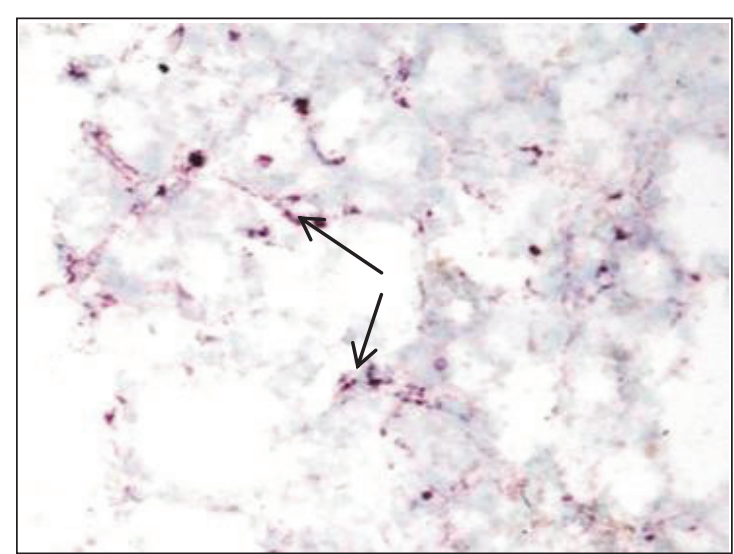

Fig. 22: Section of thyroid gland of $50.8 \mathrm{~cm} \mathrm{CVRL} \mathrm{(188} \mathrm{days)}$ foetus showing intense staining for blood vessels (arrow) in the connective tissue in interfollicular spaces. vWF X400

\section{CONCLUSION}

The present findings highlighted the distribution of different histochemical moieties and process of angiogenesis in thyroid gland of buffalo during prenatal development. The progressive increase in distribution of neutral mucopolysaccharides, proteins, lipids and number and size of blood vessels reflected the physiological and development requirements during prenatal development

\section{REFERENCES}

Chayen, J., Butcher, R.G., Bitensky, L. and Poulter. L.W. 1969. A Guide of Practical Histochemistry. $1^{\text {st }}$ Ed., Oliver and Boyd, Edinburg, England.

Edward, M.J. 1965. Observations on the anatomy of the reproductive organs of cow. N.Z. Vet.J., 13: 25.

Fagman, H. and Nilsson, M. 2010. Morphogenesis of thyroid gland. Mol. Cell. Endocrinol., 323(1): 35-54.

Hajovska, K. 2002. Prenatal thyroid gland in sheep with regard to the presence of isthmus. Anat. Histol. Embryol., 31 (5): 300-302.

Luna, L.G. 1968. Manual of histological staining methods of armed forces institute of pathology. $3^{\text {rd }} \mathrm{Ed}$., McGraw Hill, New York.

Ramayya, P.J., Ahmad, M, S., Babu, A, P., Chandrasekhara Rao, T, S., and Sudhir, P, H. 2012. Histogenesis of the thyroid and parathyroid glands in buffalo. Buffalo Bull., 31(1):12-18.

Ranjan, R., Sharma, A., Singh, O. and Bansal, N. 2011. Histogenesis of thyroid gland in buffalo. Indian J. Anim. Sci. 81 (4): 377-79.

Sheehan, D. C., and Hrapchak, B. B. 1973. Theory and Practice of Histotechnology. $1^{\text {st }}$ Ed., The C V Mosby Company, Saint Louis, USA. 
